\title{
Computationally efficient methods for climate model inversion
}

\author{
Meelis J. Zidikheri ${ }^{1} \quad$ Jorgen S. Frederiksen ${ }^{2}$
}

(Received 22 October 2012; revised 17 April 2013)

\begin{abstract}
The dynamical equations of a model are used to obtain the 'forcing function', which is a representation of climate change drivers, from an observed climatic anomaly. This inversion problem is mathematically difficult because of the two-way interaction between the mean field and transient eddies; this is known as the turbulence closure problem. The first method that we explore for overcoming the closure problem involves iteratively nudging a climate simulation towards the observed climate. We demonstrate how this method is used to successfully calculate the climatic forcing function. The second method that we explore involves finding approximations to the turbulence closure problem. In this method, the transient eddy feedback term in the mean field equation is represented as a linear combination of the mean fields and a
\end{abstract}

http://journal.austms.org.au/ojs/index.php/ANZIAMJ/article/view/6266 gives this article, (C) Austral. Mathematical Soc. 2013. Published May 28, 2013, as part of the Proceedings of the 16th Biennial Computational Techniques and Applications Conference. ISSN 1446-8735. (Print two pages per sheet of paper.) Copies of this article must not be made otherwise available on the internet; instead link directly to this URL for this article. 
constant term. We demonstrate that the closure method yields a good approximation to the climatic forcing function. This forcing function is then used as an improved first estimate in the iterative method, thereby yielding a scheme that converges very quickly to the correct solution in only a few iteration steps.

Keywords: climate change attribution; geophysical fluid dynamics; nonlinear dynamics; statistical dynamics; non-equilibrium statistical mechanics; inverse modelling.

\section{Contents}

1 Introduction

2 Iterative method

$\mathrm{C} 220$

3 Closure method

$\mathrm{C} 224$

3.1 Diagonal formulation . . . . . . . . . . . . . C225

3.2 Quasi-diagonal formulation . . . . . . . . . . . .

4 Conclusion

C229

References

C231

\section{Introduction}

Climate change attribution is a contemporary problem of great significance in climate science. The most widely used method for this purpose is 'optimal fingerprinting' which is a statistical method that estimates the amplitudes of externally forced signals in observations [1]. This method relies on the responses of atmospheric models to external forcing, which must specified a priori. We pursue a novel dynamical approach to the climate change 
attribution problem by calculating the external forcing directly from the equations of motion, given a particular climatic perturbation. This amounts to an inversion method and enables us to overcome the problem of specifying forcing mechanisms a priori, unlike the statistical method.

We pursue a computationally efficient approach in which the forcing is calculated from the mean field perturbation, without resorting to explicitly employing the transients corresponding to each particular perturbation, unlike the approach of Corti et al. [2], for example. Previously, such attempts used the fluctuation-dissipation theorem (FDT). As proposed by Leith [3] in the atmospheric context, the FDT enables the calculation of the model mean field response function from the time-lagged statistics of the transients. It was applied, with reasonable success, to atmospheric models of varying complexity $[4,5,6]$. However, the FDT method does not consistently produce good results at all geographical locations for particular flow configurations [6].

In this study we do not employ the FDT; rather, we seek to overcome the closure problem using two different techniques. The first technique uses an iterative procedure to progressively relax a climate simulation towards an observed climatic value. Starting from an initial estimate, the forcing function is adjusted at each iteration step by a term that reduces the error between the observed and simulated climate states. Provided the scheme converges, after several iterations this technique yields a climatic forcing function that is consistent with the observed climate. Although straighforward, this iterative technique does depend on a good initial estimate of the forcing function. Without a good initial estimate the number of iterations required might not be feasible if the climate model in question is very computationally intensive.

For the second technique we directly compute the forcing function from the statistical equations of motion. This problem is a difficult one because of the two-way interaction between the mean field and transient eddies, leading to the turbulence closure problem. We find approximations to the closure problem by using ideas from statistical dynamical closure theories such the quasi-diagonal direct interaction approximation (QDIA) and self 
energy (SE) closures $[7,8,9]$ as well as the phenomenology of quasi-geostrophic turbulence [10]. Computationally, this technique is used to obtain an initial estimate of the climatic forcing which is then further improved by using the iterative technique. Therefore, by combining the two techniques we obtain a methodology that is very computationally efficient and accurate.

In Section 2 we outline the iterative method and use it to simulate the January 1949-1968 climate state. We then introduce an idealised thermal source perturbation and study the efficacy of the iterative method in reproducing this source. In Section 3 we describe the diagonal closure formulation and apply it to the reconstruction of the thermal source. We then introduce an improved version of the closure, the quasi-diagonal formulation, and use it to reconstruct the same source. We also use the closure based estimate as an initial estimate for the iterative method and show that it makes the latter more computationally efficient.

\section{Iterative method}

Let a climate model be represented by

$$
\frac{\partial}{\partial t} \chi^{j}(t)=I^{j}(t)+f^{j},
$$

where $x^{j}$ is a climate variable with the superscript $j$ defining different variables such as streamfunction, wind or temperature; $\mathrm{I}^{j}$ is generally complex and represents internal couplings of the fields and processes such as rotation, dissipation, effect of bottom topography and subgrid-scale parametrisations; and $f^{j}$ is a forcing function which describes the effect of external drivers such as solar radiation, greenhouse gases and aerosols. It is assumed that the time dependence of the forcing functions occurs over a much longer timescale than typical model fluctuations and therefore the forcing functions are considered to be time independent. This is equivalent to relaxing the model to some equilibrium value, which is a common procedure in climate 
studies [12]. We use a simple model of the climate defined by the two level quasi-geostrophic equations. These equations provide a good description of midlatitude dynamics and we previously described the details [11].

To obtain the simulated climate state from the system of equations we perform some averaging procedure represented by angled brackets. The simulated climate is thus represented by $\left\langle x^{j}\right\rangle$. Starting from an initial estimate of the forcing function, $\mathrm{f}_{0}^{j}$, we obtain subsequent iterates by adding a term that is proportional to the difference between the observed climate state and the simulated climate state,

$$
f_{i+1}^{j}=f_{i}^{j}+\gamma \kappa\left(\bar{x}^{j}-\left\langle x_{i}^{j}\right\rangle\right),
$$

where the subscript $i$ is the iteration step, $\bar{x}^{j}$ is the observed climate state, $\left\langle x_{i}^{j}\right\rangle$ is the climate state of the simulation at the ith iteration step, $\kappa^{-1}$ is a fixed model parameter that controls the time scale of the model fluctuations [11], and $\gamma$ is a parameter that controls the rate of convergence of the iteration scheme. When the error $\bar{\chi}^{j}-\left\langle x_{i}^{j}\right\rangle$ is small enough, as determined by some suitable tolerance value, then the scheme has converged and $f_{i}^{j}$ is the forcing function corresponding to the observed climatic state.

Firstly, we applied the iterative method to calculate the forcing function corresponding to the basic January 1949-1968 climate state. We used the two level quasi-geostrophic model, which is described in detail by Zidikheri and Frederiksen [11]. The model was run to a statistical steady state (at which the model statistics are time independent) and sampled, by taking the time average, for a period of 200 days (10,000 timesteps) for several iterations and then sampled over a longer period of 2000 days (1,000,000 timesteps) in the final few iterations. We used the observed climate state scaled by $\kappa$, with $\mathrm{K}^{-1}$ being 10 days, as the initial estimate of the forcing function, and set $\gamma=1$. We found that after 24 iterations the pattern correlation (as defined by Namias et al. [13], for example) between the simulated and observed zonal wind data was around 0.99 , which indicates that the iteration scheme converged. A similar performance was found for other model fields such as streamfunction and vorticity. We also found that the value $\gamma=1$ is almost 
optimal; smaller values lead to slower convergence while larger values lead to less accurate solutions.

In climate change science it is often the small perturbations to the basic states that are of interest. We therefore introduce an idealised perturbation to the basic climate state in the form of a conical thermal source located at $165^{\circ} \mathrm{W} 40^{\circ} \mathrm{N}$ (an arbitrarily chosen location in the midlatitudes of the northern hemisphere). The thermal source perturbation is equivalent to a localised baroclinic (vertical shear) wind forcing perturbation that enhances the baroclinicity southward of this location and reduces it northward, as shown in Figure 1(a). The resulting model responses are shown in Figure 1(b), for the barotropic (vertical average) zonal wind response, and Figure 1(c), for the corresponding baroclinic response. The baroclinic response has a similar pattern to the forcing function, although there are weaker baroclinic responses away from the location of the source. The barotropic response is more spread out in the zonal (east-west) direction, which is a well-known phenomenon in quasi-geostrophic turbulence [10].

The idealised forcing function in Figure 1(a) is used to compare the performance of the various inverse modelling techniques considered in this study. We conducted two experiments in reconstructing the forcing function iteratively. In the first experiment we used a short sampling time of 200 days whereas in the second experiment we used a long sampling time of 2000 days. In both experiments, the forcing function was initialised with the forcing function for the basic state (no perturbation) in the iterative process. We found that for the short sampling experiment the pattern correlation between the actual and reconstructed values initially grew rapidly with number of iterations, reaching a value of 0.9 after about 30 iterations, and then saturated after about 60 iterations with a value of 0.93 . For the long sampling experiment the pattern correlation reached a value of 0.9 after about 20 iterations and saturated after about 80 iterations with a value of 0.97 . These experiments suggest that for many practical applications it is sufficient to sample for a relatively short time (about 200 days) to get a good estimate of the forcing function pattern. 


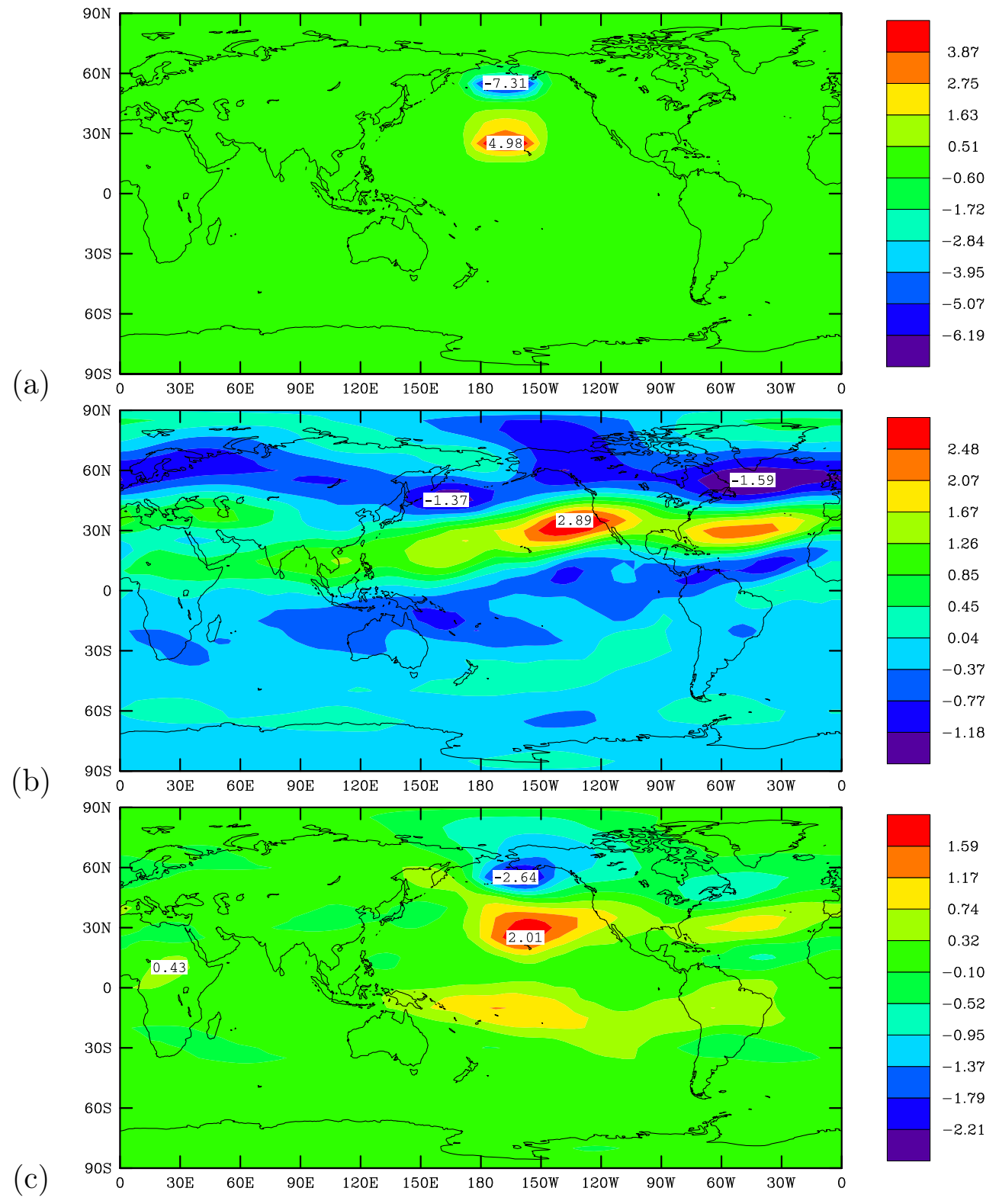

Figure 1: (a) baroclinic zonal wind forcing function perturbation (in units of $10^{-5} \mathrm{~ms}^{-2}$ ); (b) barotropic; and (c) baroclinic zonal wind climatic responses (in units of $\mathrm{ms}^{-1}$ ). 


\section{Closure method}

We construct an inverse method that is based on the statistical dynamics of the climate model. This method is specifically constructed to calculate perturbations to some basic state forcing function, unlike the iterative method which is constructed to calculate the forcing function for an arbitrary state. Assuming that the system is at steady state, it follows from (1) that the perturbed forcing function $\delta f^{j}=-\delta\left\langle\mathrm{I}^{j}\right\rangle$. Also, $\delta\left\langle\mathrm{I}^{j}\right\rangle=\delta\left\langle\mathrm{L}^{j}\right\rangle+\delta\left\langle\mathrm{N}^{j}\right\rangle+\delta\langle\mathrm{T}\rangle$, where $\left\langle\mathrm{L}^{j}\right\rangle$ consists of terms linearly dependent on the mean field, $\left\langle\mathrm{N}^{j}\right\rangle$ consists of terms nonlinearly (effectively quadratically) dependent on the mean field, and $\langle T\rangle$ consists of terms that depend on the second order moments (variances and covariances) [11].

It is straighforward to compute the first two terms, $\left\langle\mathrm{L}^{j}\right\rangle$ and $\left\langle\mathrm{N}^{j}\right\rangle$, because they depend only on the mean field (climate state), but the last term, $\langle\mathrm{T}\rangle$, is problematic because it requires information about the variability of the climate system; this is the turbulence closure problem. The physical interpretation of $\langle T\rangle$ is that it represents the feedback of transient eddies on the mean climate state. It is the goal of this study to parametrise $\langle T\rangle$ in terms of the perturbed mean fields, thereby overcoming the closure problem.

Zidikheri and Frederiksen [11] proposed a spectral method for overcoming the closure problem that parametrises the nonlinear feedback term linearly in terms of the mean field. The coefficients in this linear model were obtained by constructing ten samples from linear combinations of the July 1949-1968 and July 1975-1994 climate state forcing functions (which were obtained by iteration). It was shown that the forcing function for the perturbed state (July 1975-1994 minus July 1949-1968) was well replicated by this linear method.

Here, we wish to go further and construct a method whose parameters are obtained by sampling an ensemble formed from simulations employing random perturbations of the basic state forcing function. This method is more widely applicable as it could be used to find the forcing function for an arbitrary 
climate perturbation. We found that it is advantageous to formulate the parametrisation in physical space, rather than in spectral space, as we are seeking to include regional effects which is easier to accomplish in physical space.

\subsection{Diagonal formulation}

The physical space analogue of the spectral formulation of Zidikheri and Frederiksen [11] for perturbed fields is

$$
\delta\left\langle T^{j}(\mathbf{r})\right\rangle=\sum_{k=1}^{J} A^{j k}(\mathbf{r}) \delta\left\langle x^{k}(\mathbf{r})\right\rangle+b^{j}(\mathbf{r}),
$$

where $\mathrm{J}$ is the number of fields in the model. For the two level quasi-geostrophic model, $j, k=1,2 ; A^{j k}$ is a $2 \times 2$ matrix and $b^{j}$ a two dimensional vector, both defined on a grid which is defined by the vector $\mathbf{r}$. The components of $A^{j k}$ and $b^{j}$ are obtained by linear regression of an ensemble of samples, constructed as described below.

We scale the basic state (January 1949-1968) forcing function by a factor $p$, and multiply all spectral components of the forcing function which have wavenumbers $\leqslant 5$ (the largest scale) by a complex number of random phase and magnitude [11]. The factor $p$ is chosen to be in the range $0.0625 \leqslant$ $p \leqslant 0.175$. This is chosen to roughly correspond to typical magnitudes of atmospheric climatic anomalies over the second half of the 20th Century. We form $\mathrm{R}$ perturbations for each value of $p$ using a random number generator and use ten different values of $p$, bringing the total number of samples in the ensemble to $10 \mathrm{R}$. We experimented with different values of $R$. On obtaining the randomly perturbed forcing functions we ran a climatological simulation to obtain the mean field $\left\langle x^{j}\right\rangle$ and the nonlinear feedback term $\left\langle T^{j}\right\rangle$ for each perturbed forcing function. The coefficients $A^{j k}(\mathbf{r})$ and $b^{j}(\mathbf{r})$ in (3) were obtained by linear regression of the ensemble members. We found that the parametrisation works best when $x^{j}$ represents the zonal velocity field. The 
physical reason for this is that the nonlinear feedback term $\mathrm{T}^{j}$ is dependent on the transients, and the transients in turn are dependent on the (baroclinic) zonal velocity field. The velocity field is therefore a good predictor of the spatial variation of the transient feedback term.

We repeated the test function reconstruction experiments of the previous sections using the diagonal grid parametrisation. We found that the pattern correlation between the reconstructed forcing function and the actual forcing function increased from 0.67 with $R=1$, corresponding to 10 ensemble members, to 0.73 with $R=3$, corresponding to 30 ensemble members. Increasing the value of $R$ beyond 3 does not lead to a significant increase in the pattern correlation. The reconstructed forcing function, using 30 ensemble members, is shown in Figure 2(b). This is to be compared to the reconstructed forcing function of Figure 2(a) in which the perturbed nonlinear feedback term is assumed to be zero, which is equivalent to assuming that the basic state transients remain invariant to climatic perturbations, giving a pattern correlation of only 0.57 . The improved pattern correlation indicates that the parametrisation in (3) plays an important role in the successful reconstruction of the forcing function. These results demonstrate that the parametrisation in (3) provides a good approximation to the turbulence closure problem. Furthermore, because the samples forming the ensemble are random, this parametrisation is expected to work well for a wide variety of perturbations of the July 1949-1968 climatic basic state.

\subsection{Quasi-diagonal formulation}

The diagonal formulation of the previous section provides a good approximation to the closure problem. However, we show in this section that this formulation may be further improved. The transients (and hence the nonlinear feedback) at a given location depend not only on the baroclinicity at that location but also on the baroclinicity at other locations, especially neighbouring locations. This non-local interaction needs to be taken into account when 
(a)

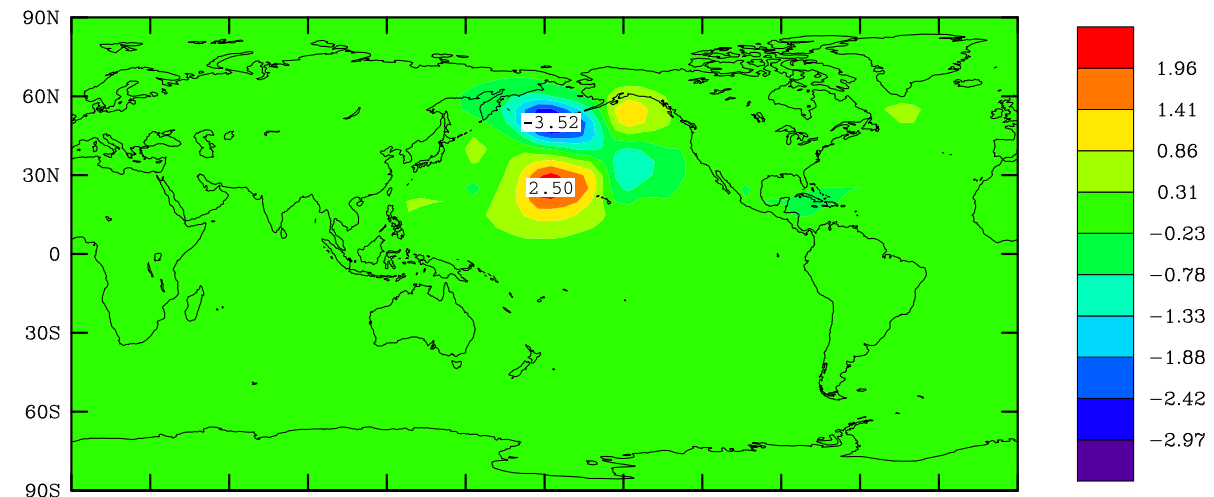

(b)
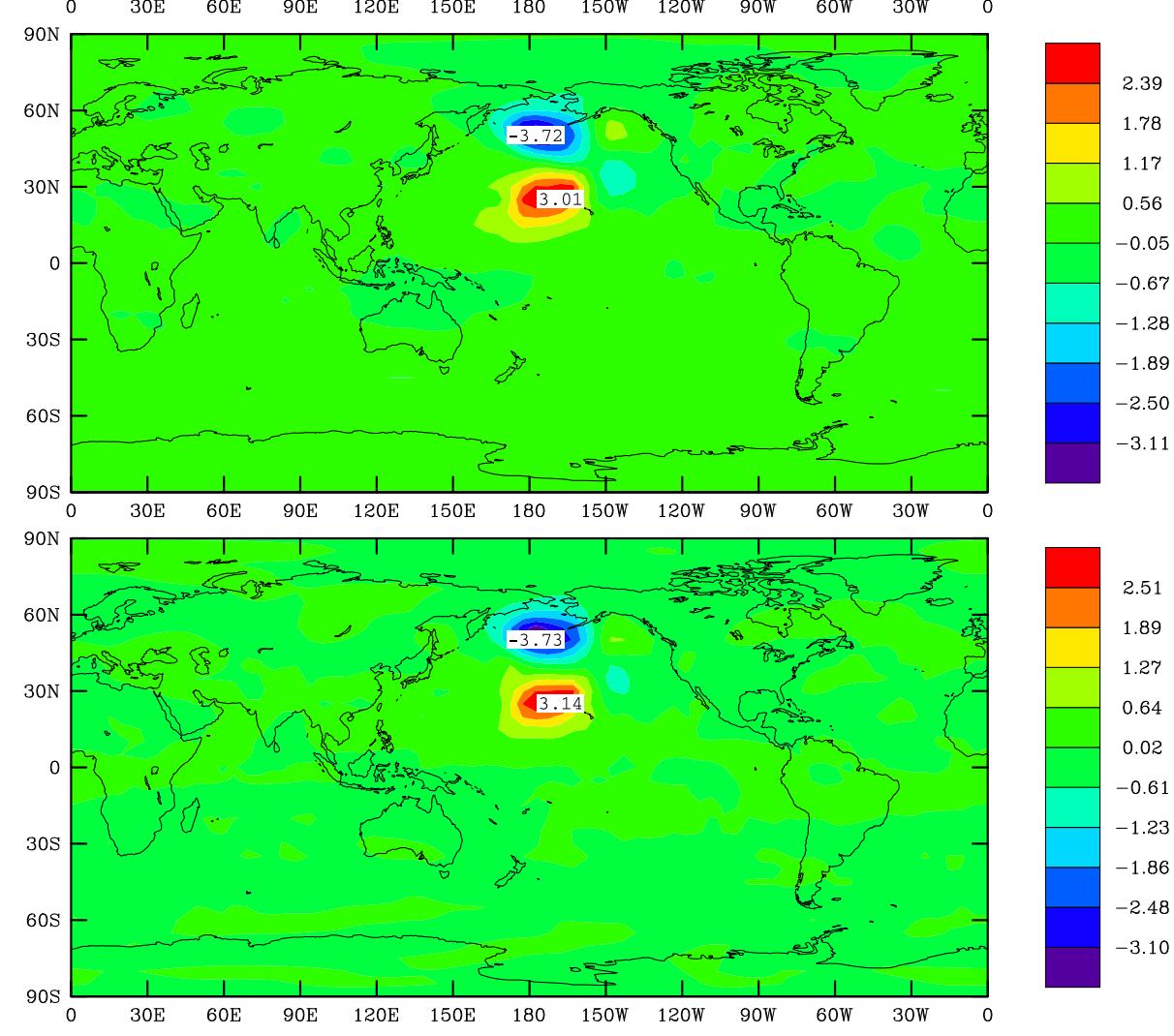

Figure 2: Reconstructed baroclinic zonal wind forcing function perturbation (in units $10^{-5} \mathrm{~ms}^{-1}$ ) using (a) no feedback term, (b) diagonal parametrisation, and (c) quasi-diagonal parametrisation of feedback term. 
formulating a closure scheme. Formally, we generalise equation (3) and the problem becomes one of determining the coefficients in

$$
\delta\left\langle\mathrm{T}^{\mathrm{j}}(\mathbf{r})\right\rangle=\sum_{\mathrm{k}=1}^{\mathrm{J}} \sum_{\mathbf{r}^{\prime}} A^{\mathrm{jk}}\left(\mathbf{r}, \mathbf{r}^{\prime}\right) \delta\left\langle\chi^{\mathrm{k}}\left(\mathbf{r}^{\prime}\right)\right\rangle+\mathrm{b}^{\mathrm{j}}(\mathbf{r}),
$$

where now, at a location $\mathbf{r}$, we have to take into account the dependence of the feedback term on the fields at other locations, $\mathbf{r}^{\prime}$. In other words, we need to also take into account the (spatially) non-diagonal elements of the covariance matrix $A^{j k}\left(\mathbf{r}, \mathbf{r}^{\prime}\right)$. It is not feasible to calculate the full covariance matrix with the number of samples at our disposal (10-100) so we resort to alternative means to estimate the effect of the off-diagonal contributions. We formulate the problem in the quasi-diagonal form

$$
\delta\left\langle T^{j}(\mathbf{r})\right\rangle=\sum_{k=1}^{J} A^{j k}(\mathbf{r}) \delta\left\langle x^{k}(\mathbf{r})\right\rangle+\sum_{k=1}^{J} B^{j k}(\mathbf{r}) \delta\left\langle\bar{x}^{k}(\mathbf{r})\right\rangle+b^{j}(\mathbf{r}),
$$

where $A^{j k}(\mathbf{r}), B^{j k}(\mathbf{r})$ and $b^{j}(\mathbf{r})$ are determined by linear regression as before. In equation (5) we defined a new field

$$
\bar{\chi}^{\mathrm{k}}(\mathbf{r})=\sum_{\mathbf{r}^{\prime} \in \mathcal{R}} \chi^{\mathrm{k}}\left(\mathbf{r}^{\prime}\right),
$$

where $\mathcal{R}$ is a region defined by the set of points $\left(\lambda^{\prime}, \phi^{\prime}\right)=\mathbf{r}^{\prime},(\lambda, \phi)=\mathbf{r}$ such that $\left|\lambda-\lambda^{\prime}\right| \leqslant \lambda_{m},\left|\phi-\phi^{\prime}\right| \leqslant \phi_{m}$, and $\lambda_{m}, \phi_{m}$ are values chosen to minimize the least-squares linear regression error. We found empirically that $\lambda_{\mathrm{m}}=\phi_{\mathrm{m}}=45^{\circ}$ give optimal results. This amounts to calculating a regional average of the mean fields at a given location. It has the effect of including the average effect of all the mean fields in the vicinity of the location $\mathbf{r}$ on the nonlinear feedback term at that location. However, the precise coupling between the feedback term at the location $\mathbf{r}$ and the mean fields at other locations $\mathbf{r}^{\prime}$ is lost. Nevertheless, we expect that these mean field averages will lead to an improvement in the purely diagonal formulation of equation (3), which only takes into account the coupling between the fields at the location $\mathbf{r}$. 
The reconstructed forcing function using the quasi-diagonal form of the parametrisation is shown in Figure 2(c), again using 30 ensemble members. The pattern correlation of the reconstructed forcing function with the actual forcing function is an improved value of 0.8 , when compared to the value of 0.73 obtained with the diagonal formulation. Both the diagonal and quasidiagonal closure formulations underestimate the actual forcing function peak values. It is possible to further improve the results obtained with the closure methods by using them as initial values in the iterative method. Because in this case the initial estimate is already very good, being in the order of pattern correlation 0.8 , the iterative method is expected to converge quickly to the correct value. This is indeed the case, as demonstrated in Figure 3. The iterative method initiated with the basic state forcing function takes about 14 iteration steps to reach the 0.8 pattern correlation level. On the other hand, the iterative method initiated with the closure based estimate reached a pattern correlation level of about 0.9 in the same number of iteration steps.

\section{Conclusion}

In this study we explored two techniques for calculating the forcing functions corresponding to climatic perturbations. In the first technique, we employed an iterative method to reconstruct a thermal source perturbation forcing function from climatic simulation data. We showed that the source is well reproduced even with relatively short sampling times, yielding a pattern correlation of 0.93. In the second technique, we used parametrised forms of the nonlinear eddy feedback term to directly calculate the perturbed forcing function using only information about the perturbed mean fields. We showed that pattern correlations of up to 0.8 could be obtained using this technique. Finally, we showed that the computational effort required in the iterative method, due to the potentially large number of iterations required to converge to the solution, might be overcome by using the estimates obtained in the 


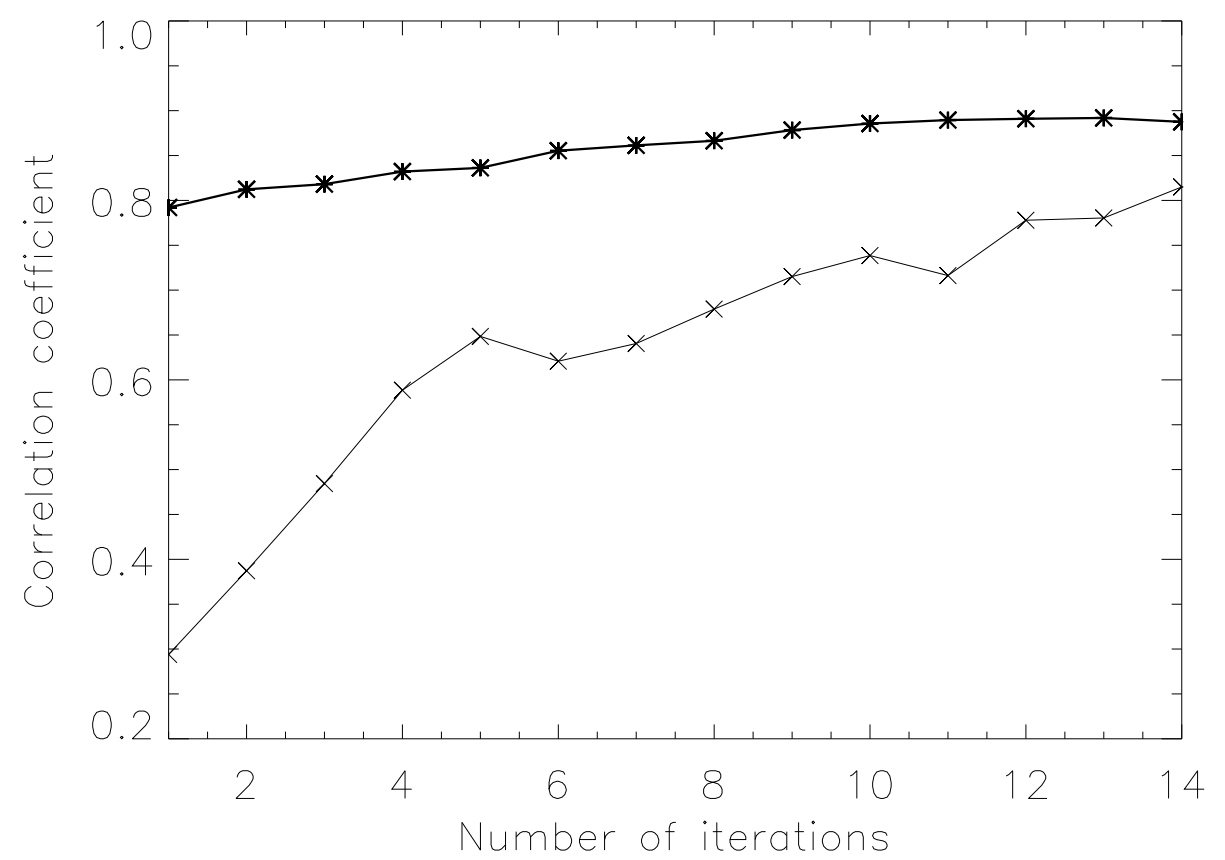

Figure 3: Variation of iteratively calculated baroclinic zonal wind forcing function pattern correlation coeffients with number of iterations for basic state initial estimate (thin line with crosses) and closure based initial estimate (solid line with asterisks).

closure based methods as an initial estimate. From a computational point of view, the iterative method then becomes a means of refining the first estimate obtained by the closure methods. The ability of the closure methods to provide good estimates of the forcing functions becomes particularly important if the forcing functions in very computationally intensive climate models, such as coupled atmosphere-ocean models, are to be successfully calculated.

Acknowledgements We acknowledge support by the West Australian Department of Environment and Conservation under the IOCI-3 program 
and the ACCSP program of the Australian Department of Climate Change and Energy Efficiency.

\section{References}

[1] G. C. Hegerl, T. R. Karl, M. Allen, N. L. Bindoff, N. Gillett, D. Karoly, $\mathrm{X}$. Zhang and F. Zwiers. Climate change detection and attribution: beyond mean temperature signals. J. Clim., 19:5058-5077, 2006. doi:10.1175/JCLI3900.1 C218

[2] S. Corti, A. Giannini, S. Tibaldi and F. Molteni. Patterns of low-frequency variability in a three-level quasi-geostrophic model. Clim. Dyn., 13:883-904, 1997. doi:10.1007/s003820050203 C219

[3] C. E. Leith. Climate response and fluctuation dissipation. J. Atmos. Sci., 32:2022-2026, 1975. doi:10.1175/1520-0469(1975)032<2022:CRAFD>2.0.CO;2 C219

[4] T. L. Bell. Climate sensitivity from fluctuation dissipation: some simple model results. J. Atmos. Sci., 37:1700-1707, 1980. doi:10.1175/1520-0469(1980)037<1700:CSFFDS>2.0.CO;2 C219

[5] A. S. Gritsun. Fluctuation-dissipation theorem on attractors of atmospheric models. Russ. J. Numer. Anal. Math. Modelling, 16:115-133, 2001. C219

[6] A. Gritsun and G. Branstator. Climate response using a three-dimensional operator based on the fluctuation-dissipation theorem. J. Atmos. Sci., 64:2558-2575, 2007. doi:10.1175/JAS3943.1 C219

[7] J. S. Frederiksen. Subgrid-scale parametrisations of eddy-topographic force, eddy viscosity, and stochastic backscatter for flow over topography. J. Atmos. Sci., 56:1481-1494, 1999.

doi:10.1175/1520-0469(1999)056<1481:SSPOET > 2.0.CO;2 C220 
[8] J. S. Frederiksen. Statistical dynamical closures and subgrid modeling for inhomogeneous QG and 3D turbulence. Entropy, 14:32-57, 2012. doi:10.3390/e14010032 C220

[9] J. S. Frederiksen. Self-energy closure for inhomogeneous turbulent flows and subgrid modeling. Entropy, 14:769-799, 2012. doi:10.3390/e14040769 C220

[10] R. Salmon. Lectures on Geophysical Fluid Dynamics, 1998. Oxford University Press. C220, C222

[11] M. J. Zidikheri and J. S. Frederiksen. Inverse method for attribution of climate change. ANZIAM J., 52:C823-C836, 2011. http://journal. austms.org.au/ojs/index.php/ANZIAMJ/article/view/3930. C221, C224, C225

[12] I. M. Held and M. J. Suarez. A proposal for the intercomparison of the dynamical cores of atmospheric general circulation models. Bull. Amer. Meteor, Soc., 75:1825-1830, 1994. doi:10.1175/1520-0477(1994)075<1825:APFTIO > 2.0.CO;2 C221

[13] J. Namias, X. Yuan and D. R. Cayan. Persistence of North Pacific sea surface temperature and atmospheric flow patterns. J. Clim., 1:682-703, 1988. doi:10.1175/1520-0442(1988)001<0682:PONPSS > 2.0.CO;2 C221

\section{Author addresses}

1. Meelis J. Zidikheri, Centre for Australian Weather and Climate Research, Bureau of Meteorology, Docklands, Victoria, Australia. mailto:m.zidikheri@bom.gov.au

2. Jorgen S. Frederiksen, Climate Adaptation Flagship, CSIRO Marine and Atmospheric Research, Aspendale, Victoria, Australia. 